- a significantly greater proportion of children with BPD compared with those preterm children without BPD had a clinically important reduction in their mean percentage predicted FEV1

- Exercise capacity is not completely normal, but the differences are more subtle than expected.

- Ct scans show linear and triangular opacities, gas trapping and mosaic perfusion, emphysema especially in severe BPD, which represent subjects with old and new BPD.

Perhaps the most important question for clinicians in the audience is "can we do anything to prevent long term sequelae and if not what should I do to explore the long term sequelae in the most efficient way?" At the end of the talk, it will be clear that preventive possibilities are scarce. However in order to improve long-term respiratory outcome and health-related quality of life of all preterm infants, efforts should be aimed at

- preventing harmful viral lower respiratory infections during the first year of life

- Reducing cigarette smoke exposure

- Parental education that may increase awareness of disease

The EFCNI White Paper on Maternal and Newborn Health and Aftercare Services states that a worldwide network of long-term follow-up research into preterm children is needed. Long-term follow-up research into societal participation of adolescents born preterm should take place. The results can be used to develop interventions for teenagers born preterm to support them in their course of life towards adulthood; for example, by assigning job or life coaches. Research of long-term medical and social treatment of preterm children is needed and also guidelines for treatment and counseling by neonatologists, paediatricians, general physicians, well baby clinics and teachers should be developed.

\section{RANDOMIZED CONTROLLED TRIAL OF DAY-CARE AND HOSPITALIZED MANAGEMENT OF SEVERE PNEUMONIA WITH SEVERE ACUTE MALNUTRITION IN CHILDREN IN BANGLADESH}

doi:10.1136/archdischild-2012-302724.0126

${ }^{1} \mathrm{H}$ Ashraf, ${ }^{1} \mathrm{NH}$ Alam, ${ }^{2} \mathrm{~N}$ Gyr. ${ }^{1}$ Centre for Nutrition and Food Security (CNFS), International Centre for Diarrhoeal Disease Research, Bangladesh (icddr, b), Dhaka, Bangladesh; 'Department of Internal Medicine, University of Basel, Basel, Switzerland

Background and aims Severe childhood pneumonia and severe acute malnutrition (SAM) require hospitalized management but inadequate pediatric beds limits hospital-care in Bangladesh. As two prospective observational studies showed that day-care facility-based management of severe pneumonia and SAM were successful as alternatives to hospitalization, a RCT was conducted.

Methods A randomized hospital (ICHSH) versus day-care (The Radda Clinic equipped with oxygen, suction, pulse oximeter, nebulizer, glucometer) comparative study was carried out to evaluate the safety and effectiveness of day-care model. Children aged 2-59 months having severe pneumonia with SAM were randomized to day-care or hospital-care. Parents brought children at 08:00 at daycare clinic and back home at 17:00 daily after receiving antibiotics, diet, micronutrients and oxygen. For hospital-care, children received similar 24-hour treatment with antibiotics, diet, micronutrients and oxygen daily. Both day-care and hospital management continued daily until improvement and discharged.

Results In total, 340 children were randomized to either day-care or hospital-care management. Successful management was possible in 136/170 [80\% (95\% CI 73.4-85.3\%)] day-care children and 144/170 [84.7\% (95\% CI 78.5-89.3\%)] hospital-care children. Of remaining 34 day-care children, 29 [17.1\% (95\% CI 12.1-23.4\%)] were referred to hospital and 5 [2.9\% (95\% CI 1.3-6.7\%)] discontinued treatment. Of remaining 26 hospital-care children, 18 [10.6\%
(95\% CI $6.8-16.1 \%)]$ were referred to specialized hospitals and 6 [3.5\% (95\% CI 1.6-7.5\%)] discontinued treatment.

Conclusion Children with severe pneumonia with SAM could be treated safely and effectively on a day-care basis at established daycare clinics, similar to hospital management, if required logistic support is available.

\section{TRENDS IN SMOKING IN PREGNANCY IN THE NETHERLANDS (2001-2010)}

doi:10.1136/archdischild-2012-302724.0127

${ }^{1} \mathrm{Cl}$ Lanting, ${ }^{1} \mathrm{JP}$ van Wouwe, ${ }^{2}$ van den Burg, ${ }^{2} \mathrm{D}$ Segaar, ${ }^{1} \mathrm{KM}$ van der Pal-de Bruin ${ }^{1}$ Netherlands Institute for Applied Scientific Research TNO, Leiden; ${ }^{2}$ Stivoro, Den Haag, The Netherlands

Background and aims Smoking in pregnancy significantly increases the risk of preterm birth and fetal growth restriction. Pregnant women are encouraged to quit smoking. Smoking in general is discouraged by antismoking laws. In the present study, we describe trends in smoking in pregnancy in the Netherlands for 2001-2010. Methods National surveys in 2001, 2002, 2003, 2005, 2007, and 2010. In well baby clinics, questionnaires were handed out to mothers with infants $\leq 6$ months.

Results Out of a total number of 28,720 questionnaires, 16,358 (57\%) were returned. Between 2001 and 2010, prevalence of smoking in pregnancy dropped by half; from $13.0 \%$ in 2001 to $6.3 \%$ in 2010 $\left(\mathrm{P}_{\text {trend }}<0.001\right)$. The odds of being a smoker was 6.3 (95\%CI 5.3-7.4) for mothers with a low education level, and 3.0 (95\% CI 2.5-3.5) for mothers with a medium education level, as compared to mothers with high education level. Independently of their educational level, mothers smoked on average five cigarettes per day while pregnant.

We observed a sharp decrease in prevalence of smoking in pregnancy from 2003 to 2005 among women with a medium or a high education level. An almost continuous, downward trend was seen among mothers with a low level education. The 2003-2005 decrease coincided with the implementation of antismoking laws in the Netherlands.

Conclusions Between 2001 and 2010, prevalence of smoking in pregnancy dropped by half. But still, in 2010, 6.3\% of Dutch pregnant women were smokers, exposing about 11,000 unborn children per year to significantly increased health risks.

\section{INTRAPERITONEAL ADMINISTRATION OF CYTIDINE 5'-DIPHOSPHOCHOLINE (CDP-CHOLINE) REDUCES HYPEROXIC LUNG INJURY IN A NEONATAL RAT MODEL OF BRONCHOPULMONARY DYSPLASIA}

doi:10.1136/archdischild-2012-302724.0128

${ }^{1} \mathrm{M}$ Cetinkaya, ${ }^{2} \mathrm{M}$ Cansev, ${ }^{1} \mathrm{C}$ Tayman, ${ }^{1} \mathrm{~F}$ Cekmez, ' $\mathrm{FE}$ Canpolat, ${ }^{2} \mathrm{M}$ Kafa, ${ }^{3} \mathrm{~S}$ Uysal, 'SU Sarıcı. ' GATA Teaching Hospital, Ankara; ${ }^{2}$ Uludağ University Medical Faculty, Bursa; ${ }^{3}$ Fatih University Medical Faculty, Ankara, Turkey

Purpose The purpose of this study was to evaluate the preventive effect of CDP-choline treatment on hyperoxic lung injury, inflammation and apoptosis in a neonatal rat model of bronchopulmonary dysplasia BPD

Methods A total of 30 newborn pups were arranged in control, hyperoxia, and hyperoxia+CDP-choline groups. Immediately after birth, pups in the control group were kept in room air containing $21 \% \mathrm{O}_{2}$ and received daily saline injections, while those in hyperoxia and hyperoxia+CDP-choline groups were exposed to $95 \% \mathrm{O}_{2}$ and received daily injections of saline and CDP-choline $(300 \mathrm{mg} / \mathrm{kg})$, respectively, throughout postnatal day 10 (P10). Histopathological scoring, radial alveolar count, lamellar body protein expression, fibrosis, proinflammatory cytokine levels, oxidant/antioxidant enzyme activities, malondialdehyde content and apoptosis were evaluated on lung samples obtained at P10. 
Results Hyperoxia induced severe lung damage as evidenced by cell infiltration, edema and fibrosis which were reduced significantly by CDP-choline treatment. Radial alveolar count and lamellar body protein expression were significantly recovered, while number of TUNEL-positive cells and active Caspase- 3 expression were decreased by CDP-choline administration. Tissue proinflammatory cytokine (IL- $1 b$, IL- 6 and TNF- $\alpha$ ) levels as well as tissue MDA content and MPO activities were reduced, whereas GSH-Px and SOD activities were preserved in hyperoxia+CDP-choline group.

Conclusions Our data show for the first time that parenteral CDPcholine administration prevents hyperoxic lung injury in a neonatal rat model of BPD. It may therefore be suggested that CDP-choline can be used as an effective therapeutic agent for prevention of BPD in case it exhibits similar effects in humans.

\section{THE PRETERM PHENOTYPE - A COGNITIVE MODEL EXPLAINS THE PROSPECTIVE RELATIONSHIP BETWEEN PREMATURITY AND MATHEMATICAL PERFORMANCE DEFICITS}

doi:10.1136/archdischild-2012-302724.0129

1J Jaekel, ${ }^{2 D}$ Wolke. 'Developmental Psychology, Ruhr-University Bochum, Bochum, Germany; ${ }^{2}$ Psychology and HSRI, Warwick Medical School, University of Warwick, Coventry, UK

Background and aims The human brain is highly susceptible to the consequences of preterm birth. Cognitive tasks vary in complexity and resource requirements, thus performance on tasks with different demands may provide information on specific cognitive differences in children related to the degree of prematurity. Mathematical performance requires simultaneous processing of information which is particularly compromised in preterm children. Our aim was to investigate the relationships between task complexity and incremental performance deficits across the full spectrum of gestational age (GA).

Methods 1,513 children ranging from 27 to 43 weeks GA were studied from birth to 8 ; 5 years as part of a prospective geographically defined longitudinal investigation of neonatal at-risk children in South Germany (Bavarian Longitudinal Study). Children's cognitive performance at 8; 5 years was measured with $\mathrm{K}-\mathrm{ABC}$ subtests Number recall, Pattern reasoning, and Calculating and with a standardized mathematics test.

Results Results were twofold:

1. Preterm children showed incremental performance deficits with increasing task complexity.

2. There was a curvilinear relationship between GA and task performance with a point of change around 32 weeks of GA.

In general, every lost week of gestation increased the adverse impact on performance. However, this relationship was strongest among tasks which required the highest cognitive workload.

Conclusions With increasing cognitive workload preterm children fall behind in test scores. This suggests that brain organisation or damage limits cognitive resources. The relationship between GA and task performance is curvilinear. Our approach may offer a theoretical foundation to scrutinize the cognitive characteristics of the preterm phenotype.

\section{EXECUTIVE FUNCTION AND IT'S IMPACT ON MATHEMATICAL UNDERACHIEVEMENT AND ATTENTION PROBLEMS IN VERY PRETERM CHILDREN}

doi:10.1136/archdischild-2012-302724.0130

${ }^{1,2} \mathrm{CSH}$ Aarnoudse-Moens, ${ }^{2} \mathrm{~N}$ Weisglas-Kuperus, ${ }^{3} \mathrm{H}$ Duivenvoorden, ${ }^{1,4} \mathrm{JB}$ van Goudoever, ${ }^{5} \mathrm{~J}$ Oosterlaan. 1Pediatrics, Emma's Children Hospital, Academic Medical
Center, Amsterdam; 2Pediatrics, Erasmus MC - Sophia Childrens Hospital; ${ }^{3}$ Erasmus MC, Rotterdam; ${ }^{4}$ Pediatrics, VU University Medical Center; 5Child Neuropsychology, VU University, Amsterdam, The Netherlands

Background and aims Despite the dramatically increased survival rates for very preterm (gestational age $\leq 30$ weeks) infants, these children's developmental outcomes remain of significant concern. A majority of non-disabled very preterm children with IOs in the average range have substantial academic and behavior problems, of which deficits in mathematics and symptoms of inattention are the most pronounced. Executive function may be an important mechanism underlying these problems, an issue only scarcely examined and aim of this study.

Methods Two-hundred non-disabled very preterm (mean age $=$ $8.2 \pm 2.5$ ) and 230 term children (mean age $=8.3 \pm 2.3$ ), all born between 1996 and 2004, were assessed with measures of mathematics/arithmetic (Dutch Pupil Monitoring System), and executive function in preschool and in primary school. Parents and teachers reported on attention problems using the Achenbach behavior questionnaires and the Disruptive Behavior Disorders Rating scales.

Results Very preterm children had significantly more mathemati$\mathrm{cal}$ and attention problems than term children (SMD's $>0.46$ ). IO significantly predicted mathematical problems $(\beta \mathrm{s}>0.15, P \mathrm{~s}<0.04)$. Executive functioning, in particular spatial span and inhibitory control, was, over and above IO, significantly predictive for mathematical problems $(\beta s=0.11, P=0.003)$ and attention problems $(\beta s>0.17$ $P$ s $<0.001)$ in primary school. Associations were stronger for very preterm than for term children.

Conclusions Very preterm birth is associated with medium-sized deficits in mathematics and attention problems. Impaired executive function and IO scores are important predictors for these adverse outcomes.

\section{PHARMACOKINETICS AND CLINICAL EFFICACY OF PHENOBARBITAL IN ASPHYXIATED NEWBORNS TREATED WITH THERAPEUTIC HYPOTHERMIA}

doi:10.1136/archdischild-2012-302724.0131

'MPH van den Broek, ${ }^{2} \mathrm{~F}$ Groenendaal, ${ }^{2} \mathrm{MC}$ Toet, ${ }^{3} \mathrm{HLM}$ van Straaten, ${ }^{4} \mathrm{JGC}$ van Hasselt, ${ }^{4}$ ADR Huitema, ${ }^{2} \mathrm{LS}$ de Vries, ${ }^{1,5} \mathrm{ACG}$ Egberts, ${ }^{1} \mathrm{CMA}$ Rademaker. ${ }^{1}$ University Medical

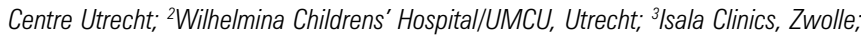
${ }^{4}$ Slotervaart Hospital, The Netherlands Cancer Institute, Amsterdam; ${ }^{5}$ Utrecht Institute for Pharmaceutical Sciences, Utrecht University, Utrecht, The Netherlands

Background and aims Therapeutic moderate hypothermia for neuroprotection in the asphyxiated newborn can influence pharmacokinetics and pharmacodynamics. If seizures occur, phenobarbital is the anticonvulsant of first choice. The aim of this study was to evaluate the effect of therapeutic hypothermia on phenobarbital pharmacokinetics and to evaluate the clinical efficacy of phenobarbital under hypothermia.

Methods Data were obtained from a prospective study in two Dutch level III NICUs (SHIVER-study). Term born newborns with criteria of perinatal asphyxia and encephalopathy were included. Therapeutic hypothermia $\left(33.5^{\circ} \mathrm{C}\right)$ was started within 6 hours after birth and was maintained for 72 hours. Pharmacokinetic modelling was performed using NONMEM.

Results In total, 31 term-born newborns were included of which 87 plasma samples were obtained (69 samples during the hypothermic phase). Based on a one-compartmental model with allometric relationships, clearance and distribution volume were estimated at $17.2 \mathrm{~mL} / \mathrm{h} / 3.5 \mathrm{~kg}$ and $3450 \mathrm{~mL} / 3.5 \mathrm{~kg}$ respectively. No relationship between hypothermia and pharmacokinetic parameters was identified. Overall, $66 \%$ of all neonates demonstrated sufficient seizure control with phenobarbital monotherapy, even though $69 \%$ of all measured concentrations were below $20 \mathrm{mg} / \mathrm{L}$. In $88 \%$ of neonates 